\title{
On Existence and Behaviour of Asymptotically Flat Solutions to the Stationary Einstein Equations
}

\section{Oscar Reula}

Max-Planck-Institut für Physik und Astrophysik, Max-Planck-Institut für Astrophysik, Karl-Schwarzschild-Strasse 1, D-8046 Garching bei München, Federal Republic of Germany

\begin{abstract}
We show existence and uniqueness of asymptotically flat solutions to the stationary Einstein equations in $S=\mathbb{R}^{3}-B_{r}$, where $B_{r}$ is a ball of radious $r>0$, when a small enough continuous complex function $\hat{u}$ on $\partial S$ is given. Regularity and decay estimates imply that these solutions are analytic in the interior of $S$ and also at infinity, when suitably conformally rescaled.
\end{abstract}

\section{Introduction}

After a considerable effort a rather clear picture describing the set of stationary, and asymptotically flat vacuum solutions to Einstein equations it is now available. Solutions are represented by a complex scalar field $u$, and a positive definite metric $g_{a b}$ on a three dimensional manifold $S[1-3]$. From the four dimensional point of view this manifold is the quotient of space-time with the set of orbits of the killing vector field defining stationarity, the metric is conformally related to the one induced by the space-time metric on $S$, and $u$ is a given functional of the norm, and the twist of the killing vector field. The equations they satisfy are,

$$
\begin{gathered}
\left(\Delta_{g}-2 R\right) u=0, \\
G_{a b}-2\left(\nabla_{(a} u \nabla_{b)} u^{*}-\left(1+4|u|^{2}\right)^{-1 / 2} \nabla_{a}|u|^{2} \nabla_{b}|u|^{2}\right) \\
-g_{a b}\left(\nabla_{c} u \nabla^{c} u^{*}-\left(1+4|u|^{2}\right)^{-1 / 2} \nabla_{c}|u|^{2} \nabla^{c}|u|^{2}\right)=0,
\end{gathered}
$$

where $G_{a b}$ is the three dimensional Einstein tensor corresponding to $g_{a b}$. We consider the following asymptotic boundary conditions $u \rightarrow 0, g_{a b}-e_{a b} \rightarrow 0$, as $r \rightarrow 0$, where $e_{a b}$ is any flat metric on $S$, and $r$ is the distance function with respect to it. Given a solution $\left(u, g_{a b}\right)$ of the above equations it is possible to reconstruct a unique, stationary, asymptotically flat, maximally extended vacuum space-time.

From local elliptic theory $[4,5]$ we know that sufficiently smooth solutions (if they exist) are in fact analytic. Furthermore assuming a stronger asymptotic decay than the one above, one can show there exists a conformal factor such that the conformally rescaled fields are also sufficiently smooth and satisfy regular elliptic equations; thus they are also analytic, even at the point representing infinity 
$[3,6,7]$. It makes sense then to characterize these solutions by their Taylor expansion at infinity, that is by a set of multipole moments $[1,2,8,9]$. It turns out that to characterize these solutions it is enough to define multipole moments only for the conformally rescaled field corresponding to $u$, that is there are no degrees of freedom associated to the three-metric $g_{a b}[10,11]$.

Unfortunately only a small set of solutions to the above equations, all of them possessing extra symmetries, are explicitly known and so until now we did not know if the asymptotic conditions assumed to obtain the above picture were generic enough so as to allow for the existence of a sufficiently large class of solutions. ${ }^{1}$ In other words we did not know how generic was this picture. In order to add weight to the above picture we shall show here the existence of a large class of solutions to the stationary Einstein equations when $S=\left\{\mathbb{R}^{3}-a\right.$ smooth ball\}. These solutions, which one would like to interpret as the exterior field of stationary compact bodies, are analytic in the interior of $S$, (even at infinity when suitably conformally transformed) and are uniquely characterized by a continuous complex function $\hat{u}$ on $\partial S$, the value of $u$ at $\partial S$, and thus by a harmonic expansion of $u$ at $\partial S$. To each member of this harmonic expansion there corresponds a unique linearized multipole moment at infinity.

\section{Main Theorem}

Existence Theorem. Fix on $\partial S$ a positive definite metric, $h_{a b}$, of constant scalar curvature. Then there exists a neighborhood of zero, $V$, in the Sobolev space ${ }^{2} \mathrm{H}_{2}(\partial S)$, such that for each $\hat{u} \in V$ there exists a unique, analytic on int $S$, and asymptotically flat solution to the stationary vacuum Einstein equations, $\left(u, g_{a b}\right)$, with $\left.u\right|_{\partial S}=\hat{u}$. The solutions, when suitably conformally transformed are analytic even at infinity, and so each of them determines a unique multipole expansion at the point at infinity.

We partition the proof in a series of lemmata. In the first we show that the stationary equations are equivalent to a reduced elliptic system. This procedure is similar to the one used to reduce the full Einstein equations to a hyperbolic one [12-14].

Reduction Lemma. Fix a flat metric, $e_{a b}$, in $S$ in such a way that it induces $h_{a b}$ on $\partial S$. Then the stationary vacuum Einstein equations, Eqs. $(1,2)$, with the boundary condition, $\left.u\right|_{\partial S}=\hat{u}$, for $\left(u, g_{a b}-e_{a b}\right) \in H_{5 / 2,-3 / 4}(S)^{3}, \hat{u} \in H_{2}(\partial S)$, small enough, are equivalent to the following reduced system for $\left(u, \phi^{a b}\right)$ :

$$
\begin{gathered}
E\left(u, \phi^{a b}\right):=\Delta_{g} u+\left(\text { terms in } \nabla_{c} \phi^{d e}, \phi^{d e}, \nabla_{c} u, u\right)=0, \\
E^{a b}\left(u, \phi^{a b}\right):=g^{c d} \nabla_{c} \nabla_{d} \phi^{a b}+\left(\text { terms in } \nabla_{c} \phi^{d e}, \phi^{d e}, \nabla_{c} u, u\right)=0, \\
\left.u\right|_{\partial S}-\hat{u}=0,\left.\quad \phi\right|_{\partial S}=0,\left.\quad \sigma_{a b}\right|_{\partial S}=0, \\
\left.\dot{\psi}\right|_{\partial S}=0,\left.\quad \hat{\psi}^{b}\right|_{\partial S}=0,
\end{gathered}
$$

\footnotetext{
${ }^{1}$ For a sufficiently large class of solutions we mean one containing all solutions corresponding to physical bodies in equilibrium occupying acompact region of space

${ }^{2}$ Proper definitions of all the spaces we are using, as well as a list of their properties can be found in [15]

${ }^{3}$ The differentiability and decay indices used for the weighted Sobolev spaces are not necessarily the sharpest ones, but rather are taken for definiteness and convenience
} 
where $\phi^{a b}:=\sqrt{g} g^{a b}-e^{a b}$, with $\sqrt{g}$ given by $\varepsilon_{a b c d}\left(g_{e f}\right)=\sqrt{g} \varepsilon_{a b c d}\left(e_{e f}\right), E^{a b}$ is obtained by setting, in the expression for $G^{a b}$ in terms of $\phi^{a b}$ the combination $\psi^{b}:=\nabla_{a} \phi^{a b}$ to zero, $\nabla_{a}$ is the covariant derivative associated to $e_{a b}$,

$$
\begin{gathered}
\phi:=e_{a b} \phi^{a b},\left.\quad \sigma_{a b}\right|_{\partial S}:=\left.\left(h_{a c} h_{b d} \phi^{c d}-\frac{1}{2} h_{a b} h_{c d} \phi^{c d}\right)\right|_{\partial S}, \\
\left.\dot{\psi}\right|_{\partial S}:=\left.\left(n^{a} \nabla_{a}\left(n_{b} \psi^{b}\right)\right)\right|_{\partial S},
\end{gathered}
$$

where $n^{a}$ is the exterior normal to $\partial S$, and $\left.\hat{\psi}^{b}\right|_{\partial S}:=\left.\left(\psi^{a} h_{a}^{b}\right)\right|_{\partial S} \cdot{ }^{4}$

Proof. First we show that any small enough solution to the reduced system is also a solution to the stationary Einstein equations. Let $\left(u, \phi^{a b}\right)$ be a solution to the reduced system and consider,

$$
\nabla_{a} E^{a b}\left(u, \phi^{c d}\right)=0 .
$$

It is easy to see that this is an elliptic linear second order equation for $\psi^{b}$, $L_{\left(u, \phi^{c d}\right)} \psi^{b}=0$, which is continuous as a map from $\left(u, \phi^{c d}\right) \in H_{5 / 2,-3 / 4}(S)$, $\psi^{b} \in H_{3 / 2,1 / 4}(S)$, to $H_{-1 / 2,9 / 4}^{\cdot}(S)$, and which reduces to the flat Laplacian when $u=\phi^{a b}=0$. But injectivity is a continuous property for elliptic operators, and since the flat Laplacian is injective for the boundary conditions considered $\left(\left.\dot{\psi}\right|_{\partial S}=0,\left.\hat{\psi}^{b}\right|_{\partial S}=0\right),{ }^{5}$ there exists $\varepsilon>0$ such that if $\left\|\left(u, \phi^{c d}\right)\right\|_{5 / 2,-3 / 4}<\varepsilon$, then $\psi^{b}=0$. Thus for these solutions both systems are identical and so they also solve the stationary Einstein equations.

Second we show that for any metric $g_{a b}$ close enough to some flat metric, $e_{a b}$, there exists another flat metric $\tilde{e}_{a b}$ with respect to which $\psi^{b}\left(g_{c d}\right)=\left.\phi\right|_{\partial S}=\left.\sigma_{a b}\right|_{\partial S}=0$. Thus, if $\left(u, g_{a b}\right)$ satisfies the stationary Einstein equations it also satisfies the reduced ones. Instead of looking for $\tilde{e}_{a b}$, which is related by a diffeomorphism to $e_{a b}$, we diffeomorphically transform $g_{a b}$, keeping $e_{a b}$ fixed. Such diffeomorphism, $d$, must satisfy,

$$
0=G\left(d, g_{a b}\right):=\left(\begin{array}{c}
\nabla_{a}^{*} \phi^{a b} \\
\left.* \phi\right|_{\partial S} \\
D_{a}^{*} \sigma^{a b}
\end{array}\right),
$$

where $* \phi^{a b}:=\sqrt{* g^{*}} g^{a b}-e^{a b}$, with ${ }^{*} g^{a b}$ the diffeomorphically transformed $g^{a b}$, and $D_{a}$ is the covariant derivative on $\partial S$ associated to $h_{a b}{ }^{6}$ We now use a corollary of the implicit function theorem on Banach spaces $[18,19]$ to assert, given any $g_{a b}$ in a small enough neighborhood of $e_{a b}$, the existence of diffeomorphisms, $d$, satisfying $G\left(d, g_{a b}\right)=0$.

Corollary. Let $X, Y, Z$ be Banach spaces, $U, V$ open sets of $X$, and $Y$ respectively, and $G: V \times U \rightarrow Z$ a $C^{1}$ function. Assume there exist $x_{0} \in U$, and $y_{0} \in Y$, such that

\footnotetext{
${ }^{4}$ Where needed we have extended $n^{a}$, and therefore $h_{a b}$, into a neighborhood of $\partial S$ on $S$ using the geodesic equation

${ }^{5}$ See Appendix and [15]

${ }^{6}$ This is equivalent to the existence of a unique, up to translations and rotations, harmonic coordinate system, once $\partial S$ is given. See also $[6,16,17]$
} 
$G\left(y_{0}, x_{0}\right)=0$, and $D_{1} G\left(y_{0}, x_{0}\right): Y \rightarrow Z$, the differential of $G$ with respect to the first argument is surjective and has complemented kernel. Then there exist neighborhoods $V_{x_{0}} \subset V$, and $U_{y_{0}} \subset U$ around $x_{0}$, and $y_{0}$ respectively such that for any $x \in U_{x_{0}}$ there exists at least an $y \in V_{y_{0}}$ satisfying $G(x, y)=0$.

Proof. It follows from Corollary 2.5.8 and the proof of the implicit function theorem in [18].

We consider $G\left(d, g_{a b}\right)$ as a function from $Y \times X$ to $Z$, where $Y=H_{7 / 2,-7 / 4}(S)$, $X=H_{5 / 2,-3 / 4}^{\ddot{2}}(S)$, and

$$
Z=H_{3 / 2,1 / 4}^{\cdot}(S) \times H_{2}(\partial S) \times\left\{H_{1}(\partial S) / \text { infinitesimal conformal isometries of } h_{a b}\right\} .
$$

$V$ is chosen, using that $H_{7 / 2,-7 / 4}(S) \subset C_{1 / 2}^{1}(S)$, to be a small enough neighborhood of the identity map in order to guarantee that all its elements have differentiable inverses. This function satisfies $G\left(i, e_{a b}\right)=0$, where $i$ is the identity diffeomorphism, is a differentiable function of both arguments, and its differential with respect to the first argument at $\left(d, g_{a b}\right)=\left(i, e_{a b}\right)$ is,

$$
D_{1} G_{\left(i, e_{a b}\right)} \cdot \delta d=\left(\begin{array}{c}
\Delta_{e} \xi^{b} \\
\dot{\xi}+k \xi+D_{a} \hat{\xi}^{a} \\
D_{a}\left(2 D^{(a} \hat{\xi}^{b)}-h^{a b} D_{c} \hat{\xi}^{c}\right)
\end{array}\right),
$$

where we have identified $\delta d$ with the vector field it generates, $\xi^{a}$, and $\xi=\left.n_{a} \xi^{a}\right|_{\partial S}$, $\dot{\xi}=\left.\left(n^{c} \nabla_{c} \xi\right)\right|_{\partial S}, k=\left.\nabla_{a} n^{a}\right|_{\partial S}$, and $\hat{\xi}^{a}=\left.h^{a}{ }_{b} \xi^{b}\right|_{\partial S}$.

Thus to complete the proof we need only to show that the above linear map has complemented kernel and is surjective. This map satisfies the hypothesis of the Main Elliptic Theorem of the Appendix. Since its kernel and the one of its adjoint, $D G_{\left(i, e_{a b}\right)}^{\prime}: Y \rightarrow X^{\prime}$, consist of $C^{\infty}$ functions, it is easy to see that in the first case it consists only of the infinitesimal isometries of $e_{a b}$, since this space is finitedimensional it is complemented, and in the second only of the infinitesimal conformal isometries of $h_{a b}$, which have been factored out, but then Range $D G_{\left(i, e_{a b}\right)}=Y$, and so surjectivity is also asserted.

Finally we show that if $\hat{\sigma}_{a b} \in H_{2}^{*}(\partial S)$ is trace and divergence free, then $\hat{\sigma}_{a b}=0$. To see this, conformally transform $h_{a b}=\Omega^{2} f_{a b}, \hat{\sigma}^{a b}=\Omega^{-4} \sigma^{a b}$, using $\Omega=\frac{2}{1+r^{2}}$, so that $f_{a b}$ is a flat metric in $\mathbb{R}^{2}$. Since $\hat{\sigma}^{a b}$ is trace free its divergence is invariant under the above conformal transformation, therefore $\sigma^{a b}$ is also trace and divergence free. But then in a cartesian (with respect to $f_{a b}$ ) coordinate system the components of $\sigma^{a b}$ satisfy the Laplacian equation, $\Delta_{f} \sigma^{\mu \nu}=0$, and so they are constant or grow asymptotically. In any case they always give rise to singular tensors when conformally transformed back to $\partial S$. Thus $\hat{\sigma}^{a b}$ must vanish.

Strictly speaking the diffeomorphism we have used on the second part of the above proof can move the sphere around, therefore one should take $\partial S$ to be contained in a open region where the stationary solution exists.

Note that although the tensor density $\phi^{a b}$ is background $\left(e_{a b}\right)$ dependent, the metric tensor it generates, $g_{a b}$, is not. The background metric is only an artifact to render the equations elliptic and thus to establish existence. 
We now use the fact that the reduced system is elliptic in the sense of Hörmander, $[20,21]^{7}$ which includes the Lopatinskî̌-Šapiro conditions [22, 23] for the boundary equations, to establish existence of solutions to the reduced system.

Existence Lemma. There exists $\varepsilon>0$, such that for each complex function $\hat{u}$ with $\|\hat{u}\|_{2, \partial S}<\varepsilon$ there exists a unique $\left(u, \phi^{a b}\right) \in H_{5 / 2,-3 / 4}(S)$ solution to the reduced system, Eqs. $(1,2)$.

Proof. Consider the reduced system as an implicit function, $F\left(\left(u, \phi^{a b}\right), \hat{u}\right)=0$, for $\left(u, \phi^{a b}\right)$ in terms of $\hat{u}$. As a function from

$$
Y \times X:=\left(H_{5 / 2,-3 / 4}(S) \times H_{5 / 2,-3 / 4}^{\ddot{*}}(S)\right) \times H_{2}(\partial S)
$$

to

$$
Z:=H_{1 / 2,5 / 4}(S) \times H_{1 / 2,5 / 4}^{\ddot{\prime}}(S) \times H_{2}(\partial S) \times H_{2}(\partial S) \times H_{2} \ddot{(}(\partial S) \times H_{0}(\partial S) \times H_{1}(\partial S)
$$

it is differentiable and furthermore $F(0,0), 0)=0$. Thus if we could show that $D_{1} F_{(0,0), 0)}:\left(\delta u, \delta \phi^{a b}\right) \in Y \rightarrow Z$, the differential of $F$ with respect to the first entry, is an isomorphism then the implicit function theorem $[18,19]$ would assert the existence of $\varepsilon>0$, and of a unique, continuously differentiable function $\left(u(\hat{u}), \phi^{a b}(\hat{u})\right)$ defined for all $\|\hat{u}\|_{2, \partial S}<\varepsilon$ such that $F\left(\left(u(\hat{u}), \phi^{a b}(\hat{u})\right), \hat{u}\right)=0$. This would complete the proof.

We first show injectivity, namely $D_{1} F_{((0,0), 0)}\left(\delta u, \delta \phi^{a b}\right)=0 \Rightarrow\left(\delta u, \delta \phi^{a b}\right)=0$.

Decomposing $\delta \phi^{a b}$ at the boundary as $\delta \phi^{a b}=\tau n^{a} n^{b}+2 n^{(a} \hat{\phi}^{b)}+\frac{\sigma}{2} \hat{h}^{a b}+\hat{\sigma}^{a b}$, with $0=\hat{\phi}^{a} n_{a}=\hat{\sigma}^{a b} n_{a}=\hat{\sigma}^{a b} h_{a b}$, we obtain

$$
0=D_{1} F_{((0,0), 0)} \cdot\left(\delta u, \delta \phi^{a b}\right)=\left(\begin{array}{c}
\Delta_{e} \delta u \\
\Delta_{e} \delta \phi^{a b} \\
\left.\delta u\right|_{\partial S} \\
\tau+\sigma \\
\hat{\sigma}^{a b} \\
D^{2}\left(\tau+\frac{\sigma}{2}\right)-\frac{k^{2}}{2}\left(\tau-\frac{\sigma}{2}\right)+\frac{k}{2} \dot{\sigma} \\
\dot{\phi}^{b}+\frac{3}{2} k \hat{\phi}^{b}+\frac{1}{2} D^{b} \sigma
\end{array}\right)
$$

\footnotetext{
${ }^{7}$ Hörmander ellipticity of the system for points not at the boundary follows from usual ellipticity. That is one considers for each one of these points the constant coefficients differential system on $\mathbb{R}^{3}$ obtained by defining its coefficients as the value of those of the principal part of the system at that point. One then shows that there is no bounded solution to the constant coefficients system of the form $u_{l}(x)=C_{l} \exp \left(i k_{j} x^{j}\right)$ with some $k_{j} \neq 0$. For points at the boundary one considers for each one of them the constant coefficients differential system on ${ }^{+} \mathbb{R}^{3}$, obtained by defining its coefficients as the value of those of the principal part of the operators at that point (now taking into account the boundary operators!). One then shows that there is no bounded solution to it of the form $u_{l}=C_{l} \exp \left(i \hat{k}_{j} \hat{x}^{j}\right) \omega(\tau)$, with some $\hat{k}_{j} \neq 0$, where $\tau$ is the coordinate defining ${ }^{+} \mathbb{R}^{3}$ as $\tau \leqq 0$, and the $\hat{x}^{j}$ are normal coordinates to $\tau$
} 
Using the first and third lines of the above equation we get

$$
0=\int_{S} \delta u \Delta_{e} \delta u d V_{e}=\int_{S} \nabla_{c} \delta u \nabla^{c} \delta u d V_{e} .
$$

Therefore $\delta u=$ cte, but the constant functions are not in $H_{5 / 2,-3 / 4}(S)$ and so $\delta u=0$. Similarly the trace of the second line, and the fourth line imply $e_{a b} \delta \phi^{a b}=0$, and so $\dot{\sigma}+\dot{\tau}=0$. Thus the last two lines become,

$$
\frac{1}{2} D^{2} \tau-\frac{3}{4} k^{2} \tau-\frac{k}{2} \dot{\tau}=0, \quad \dot{\hat{\phi}}^{b}+\frac{3}{2} k \hat{\phi}^{b}-\frac{1}{2} D^{b} \tau=0 .
$$

Using these two equations we now obtain,

$$
0=\int_{S} \delta \phi_{a b} \Delta_{e} \delta \phi^{a b} d V_{e}=\int_{S} \nabla_{c} \delta \phi_{a b} \nabla^{c} \delta \phi^{a b} d V_{e}+\int_{\partial S}\left(\frac{3}{2} k \hat{\phi}_{a} \hat{\phi}^{a}+\frac{1}{k} D_{a} \tau D^{a} \tau+\frac{k}{4} \tau^{2}\right) d V_{h}
$$

Since $k>0$ the right-hand side is positive definite, and so we conclude $\delta \phi^{a b}=0$ which asserts injectivity.

Second we prove surjectivity. Note that $D_{1} F_{((0,0), 0)} \cdot\left(\delta u, \delta \phi^{a b}\right)$ satisfies the hypothesis of the Main Elliptic Theorem of the Appendix, (in particular Hörmander ellipticity), thus it has an index, and it only depends on the principal parts of the operators. We now prove that this index vanishes. This together with the above injectivity result implies that the co-dimension of the range is zero, and so surjectivity. To find the index we consider a family of nested spheres (with respect to the flat metric $e_{a b}$ ) and decompose as before $\Delta_{e} \delta \phi^{a b}$ into equations for $\tau$, $\sigma, \hat{\phi}^{b}$, and $\hat{\sigma}^{a b}$, now defined for the whole family of spheres. Since the index is an invariant - it only depends on the principal part of the equations - one can continuously distort the equations without changing it. We distort them by setting to zero $k$, the value of the scalar curvature of the spheres, in several places where it appears in such a way as to decouple the equations. ${ }^{8}$ It is easy to show then that the resulting map, which consists of several decoupled Laplacians is an isomorphism, and therefore the vanishing of its index.

We now study regularity for the solutions whose existence has been asserted.

Regularity Lemma. Let $S^{\prime}$ be strictly contained in $S$. Then $\left(u, \phi^{a b}\right) \in C^{\omega}\left(S^{\prime}\right)$, and therefore $\left(u, g_{a b}\right) \in C^{\omega}\left(S^{\prime}\right)$. Furthermore there exists an analytic conformal factor, $\Omega$ approaching zero asymptotically as $r^{2}$ such that $\left(\tilde{u}, \tilde{g}_{a b}\right)=\left(\Omega^{-1 / 2} u, \Omega^{2} g_{a b}\right)$ can be extended analytically to the conformally completed space, that is when the point at infinity is added to $S^{\prime}$.

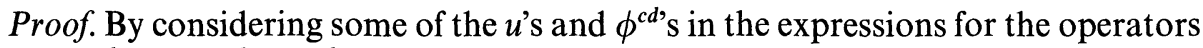
$E\left(u, \phi^{c d}\right)$, and $E^{a b}\left(u, \phi^{c d}\right)$ as coefficients we arrive to a coupled, linear elliptic system for the remaining $u$ 's and $\phi^{c d}$ 's. If $\left(u, \phi^{a b}\right) \in H_{s,-3 / 4}\left(S^{\prime}\right)$ then such system approaches $\Delta_{e}$ with differentiability $s$, and rate -1 . Furthermore since $C_{i}^{j}=0$ we can apply the Regularity Theorem of the Appendix to conclude that $\left(u, \phi^{a b}\right) \in H_{s+1,-3 / 4}\left(S^{\prime}\right)$. Starting with $s=\frac{5}{2}$ and iterating the procedure we conclude $\left(u, \phi^{a b}\right) \in H_{s^{\prime},-3 / 4}\left(S^{\prime}\right)$ for

${ }^{8}$ One is not deforming the spheres into planes, which would result in a discontinuous distortion of the Laplacian, but rather setting to zero some terms not in the principal parts of the differential operators 
any $s^{\prime}$, and so $\left(u, \phi^{a b}\right) \in H_{\infty,-3 / 4} C C_{3 / 4-\varepsilon}^{\infty}\left(S^{\prime}\right), \varepsilon>0$. Local analyticity follows now from this result and Morrey's theorem $[4,5]$. Analyticity at infinity follows from the above results and the work of Beig and Simon [10]. Although Beig and Simon assumed a faster decay at infinity than the one we have so far obtained here it is not difficult to see that their results are still valid assuming this slower decay. Alternatively one can, by writing the reduced equations as,

$$
\begin{aligned}
\Delta_{e} u & =F\left(u, \nabla_{c} u, \phi^{e f}, \nabla_{c} \phi^{e f}, \nabla_{c} \nabla_{d} \phi^{e f}\right), \\
\Delta_{e} \phi^{a b} & =F^{a b}\left(u, \nabla_{c} u, \phi^{e f}, \nabla_{c} \phi^{e f}, \nabla_{c} \nabla_{d} \phi^{e f}\right),
\end{aligned}
$$

by using the decay estimates obtained see that $\left(F, F^{a b}\right) \in C_{7 / 2-\varepsilon}^{\infty}$, and by applying the Asymptotic Behaviour Theorem of the Appendix, conclude that $\left(u, \phi^{a b}\right)$ do in fact decay as it was assumed by Beig and Simon.

This completes the proof of the Main Theorem.

\section{Discussion}

We have asserted the existence and uniqueness of solutions to the stationary vacuum Einstein equations, when a sufficiently small - with respect to a Sobolev norm - complex function, $\hat{u}$, on a sphere is given. Thus the freedom on the boundary data is the same as the one available from a multipole moment expansion at infinity. It is clear that each $\hat{u}$ giving rise to a solution determines a unique multipole set. Does there exist a norm on the multipole set such that each small enough multipole set gives rise to a unique solution to the stationary equations and thus determines a unique $\hat{u}$ ?

One would expect that it would be possible to assert existence not only for solutions close to Minkowski space-time but also for those close to Schwarzschild space-times of arbitrary mass. This is indeed the case, since by applying a constant conformal transformation to any of our solutions with nonvanishing mass we obtain solutions with arbitrarily large mass ${ }^{9}$. This corresponds to letting the sphere $\partial S$ get bigger and bigger, i.e. having smaller and smaller constant curvature, while keeping the same $\hat{u}$.

Along the same line as above it should be possible to establish existence for the interior problem, namely when $S$ is taken to be a ball, or the space within two concentric spheres. This should be relevant in order to perform an analysis similar to the one in [14], but for quantum cosmologies.

Acknowledgement. I thank Bernd Schmidt for calling my attention to the problem here treated, and for several discussions and ideas, and the referee for pointing out several places of the manuscript needing clarification or correction.

\section{Appendix}

Here we list several definitions and theorems used in this work, which although known are very difficult to find in the literature.

\footnotetext{
${ }^{9}$ I thank Bernd Schmidt for this argument
} 
Definition (Bartnik [17]) $)^{10}$. A linear elliptic operator in an $n$ dimensional asymptotically flat manifold is said to have differentiability, $s>\frac{n}{2}$, and to be asymptotic to, $\Delta_{e}$, for some flat metric $e_{a b}\left(\right.$ at rate $\left.\tau>-\frac{n}{2}\right)$, if it can be expressed as,

$$
P(u)=A_{i}^{j a b} \nabla_{a} \nabla_{b} u_{j}+B_{i}^{j b} \nabla_{b} u_{j}+C_{i}^{j} u_{j},
$$

with $\nabla_{a}$ the covariant derivative associated to $e_{a b}$, and $A_{i}^{j a b}, B_{i}^{j b}$, and $C_{i}^{j}$ tensor fields satisfying,

$$
\left\|A_{i}^{j a b}-I_{i}^{j} e^{a b}\right\|_{s, \tau}+\left\|B_{i}^{j b}\right\|_{s-1, r+1}+\left\|C_{i}^{j}\right\|_{s-2, \tau+2}<\infty .
$$

Note that the Sobolev imbedding theorems imply that $A_{i}^{j a b}$ is Hölder continuous, and $\left|A^{a b}-I e^{a b}\right|=o\left(r^{-\tau-\frac{n}{2}}\right)[15]$. For this work it is enough to consider the case $\tau=-1$.

Main Elliptic Theorem. Let $u_{j} \rightarrow P_{i}^{j} u_{j}, i, j,=1, \ldots, N$ be an operator and asymptotic to $\Delta_{e}$, at rate -1 , on $S=\mathbb{R}^{3}-B_{1}$ and let $u_{j} \rightarrow B_{i}^{(k) j} u_{j}, i, j, k=1, \ldots, N$ be boundary operators on $\partial S$. Then if the coefficients of the whole system are smooth and the whole system is elliptic in the sense of Hörmander [20,21] we have;

i) The map

$$
(P, B): \prod_{j=1}^{N} H_{s, \delta}(S) \rightarrow \prod_{i=1}^{N} H_{s-2, \delta+2}(S) \times \prod_{k=1}^{N} H_{s-2-m_{k}}(\partial S),
$$

for $s>2, \delta \neq p+\frac{1}{2}, p \in Z$, and where $m_{k}$ denotes the order of $B^{(k)}$, is Fredholm. That is, $\operatorname{dim} \operatorname{Kernel}<\infty$, co-dim $\operatorname{Range}<\infty$, and $i=\operatorname{dim} \operatorname{Ker}(P, B)-\operatorname{co-dim} \operatorname{Range}(P, B)$ is an invariant.

ii) For any $u \in H_{s, \delta}(S)$,

$$
\|u-\operatorname{Ker}(P, B)\|_{s, \delta, S} \leqq C\left\{\|P(u)\|_{s-2, \delta+2, s}+\sum_{k=1}^{N}\left\|B^{(k)}(u)\right\|_{s-1 / 2-m_{k}, \partial s}\right\},
$$

where $C$ depends only on $(P, B)$, and

$$
\|u-\operatorname{Ker}(P, B)\|_{s, \delta, s}=\inf \left\{\|u-w\|_{s, \delta, s}: w \in \operatorname{Ker}(P, B)\right\} .
$$

iii) Range $(P, B)^{\perp} C C^{\infty}(S)$.

Sketch of Proof. To prove the above theorem we follow Bartnik's proof of Proposition 1.11 in [17] which establishes a similar result but for manifolds without boundaries. We depart from that proof in the following two points:

i) To prove the analog to Proposition 1.6 in [17] instead of starting with the usual local Gärding estimate, we start with the following one which reaches the boundary: There exists $C>0$ such that for all $u \in H_{s}(\bar{S}), \partial S \subset \bar{S} \subset S$, compact,

$$
\|u\|_{s, \bar{S}} \leqq C\left\{\|P(u)\|_{s-2, \bar{S}}+\sum_{k=1}^{N}\left\|B^{(k)}(u)\right\|_{s-1 / 2-m_{k}, \partial S}\right\}
$$

\footnotetext{
${ }^{10}$ Note the different convention for the Sobolev indices in [17]
} 
This estimate can be deduced following the steps on the proof of Lemma 10.5.1 in [21] where the above inequality is proven for the case $N=1$.

ii) To prove the analog to Theorem 1.10 in [17] we take the patch function equal to 1 in a compact neighborhood of $S$ containing $\partial S$.

Remark. Partial proofs of the above theorem can be found in [15, 24-27]. We suspect that the theorem still remains valid if the smoothness condition on the coefficients is relaxed, that is if only certain differentiability, $s^{\prime}>\frac{n}{2}$, where $n=\operatorname{dim} S$, is required for $P$, and that

$$
B_{i}^{(k) j}=D_{i}^{(k) j a b} \nabla_{a} \nabla_{b}+E_{i}^{(k) j b} \nabla_{b}+F_{i}^{(k) j},
$$

with $D_{i}^{(k) j a b} \in H_{s^{\prime}-1 / 2, \partial S}, E_{i}^{(k) j b} \in H_{s^{\prime}-m_{k}+1 / 2, \partial S}, \quad F_{i}^{(k) j} \in H_{s^{\prime}-m_{k}-1 / 2, \partial S}$, and $D_{i}^{(k) j a b} n_{a}$ $=E_{i}^{(k) j b} n_{b}=0$.

Regularity Theorem (Choquet-Bruhat and Christodoulou [15]). Let $P$ be an elliptic operator of differentiability $s>\frac{3}{2}$, asymptotic to $\Delta_{e}$ at rate -1 , and with $C_{i}^{j}=0$, defined in $S=\mathbb{R}^{3}-B_{1}$. Then for any $S^{\prime} \subset S, S^{\prime} \cap \partial S=\emptyset$, there exists $C>0$ such that if $u \in H_{2, \delta}(S)$, and $P(u) \in H_{s^{\prime}-2, \delta+2}\left(S^{\prime}\right), s^{\prime} \leqq s+1$, then,

$$
\|u\|_{s^{\prime}, \delta, S^{\prime}} \leqq C\left\{\|P(u)\|_{s^{\prime}-2, \delta+2, S^{\prime}}+\|u\|_{0, \delta, S^{\prime}}\right\} .
$$

The proof of the above theorem is an immediate consequence of the local elliptic theory, see for example [28], and Theorem 6.1 in [15].

Asymptotic Behaviour Theorem (Meyers [29], Beig and Simon [9]). Let $u \in C_{\varepsilon}^{\infty}\left(\mathbb{R}^{3}\right), \varepsilon>0$, such that $\Delta_{e} u:=f \in C_{q+\varepsilon}^{\infty}\left(\mathbb{R}^{3}\right), q \in N, q \geqq 3$. Then,

$$
u=\sum_{l=0}^{q-3} \sum_{m=-l}^{l} \frac{C_{l}}{r^{l+1}} Y_{l, m}(\vartheta, \varphi)+v
$$

where $v \in C_{q-2+\varepsilon}^{\infty}\left(\mathbb{R}^{3}\right)$.

Proof. Using Green's function we have,

$$
u=\int_{\mathbb{R}^{3}} \frac{f\left(\mathbf{x}^{\prime}\right)}{\left|\mathbf{x}-\mathbf{x}^{\prime}\right|} d V .
$$

To estimate $v$ one uses the following inequality valid for all $p \in N[30,31]$,

$$
\left|\frac{1}{\sqrt{1+\omega^{2}-2 \omega \xi}}-\sum_{l=0}^{p-1} \omega^{l} P_{l}(\xi)\right| \leqq \frac{2 p+1}{\sqrt{1+\omega^{2}-2 \omega \xi}} \omega^{p},
$$

and the fact that the $n^{\text {th }}$ derivative of $u$ satisfies the Poisson equation with a source decaying as $r^{-q-n-\varepsilon}$.

\section{References}

1. Geroch, R.: J. Math. Phys. 12, 918 (1971)

2. Hansen, R.O.: J. Math. Phys. 15, 46 (1974)

3. Kundu, P.: J. Math. Phys. 22, 2006 (1981)

4. H. Müller zum Hagen: Proc. Camb. Phil. Soc. 67, 415 (1970); Proc. Camb. Phil. Soc. 68, 199 (1970) 
5. Morrey, C.B.: Contributions to theory of partial differential equations. Annals of Mathematics Studies, No. 33. Princeton, NJ: Princeton U.P., 1954; Am. J. Math. 80, 198 (1958)

6. Beig, R.: Gen. Rel. Grav. 12, 439 (1980)

7. Beig, R., Simon, W.: Gen. Rel. Grav. 12, 1003 (1980)

8. Geroch, R.: J. Math. Phys. 11, 2580 (1970)

9. Beig, R., Simon, W.: J. Math. Phys. 24, 1163-1171 (1983)

10. Beig, R., Simon, W.: Proc. R. Soc. Lond. A 376, 333 (1981)

11. Beig, R., Simon, W.: Commun. Math. Phys. 78, 75-82 (1980)

12. Choquet-Bruhat, Y., York, J.W.: The Cauchy problem, general relativity and gravitation. Held, A. (ed.). London: Plenum Press 1980

13. Wald, R.: General relativity. Chicago, IL: The Univ. of Chicago Press 1984

14. Reula, O.: A configuration space for quantum gravity and solutions to the Euclidean Einstein Equations in a slab region. Class. Quant. Grav. (to appear)

15. Choquet-Bruhat, Y., Christodoulou, D.: Acta Math. 146, 129 (1981)

16. Choquet-Bruhat, Y., Marsden, J.E.: Commun. Math. Phys. 51, 283 (1976)

17. Bartnik, R.: Note the different convention for the functional spaces indices. Commun. Pure Appl. Math. 39, 661 (1986)

18. Abraham, R., Marsden, J., Ratiu, T.: Manifolds, tensor analysis, and applications. Reading, MA: Addison-Wesley 1983

19. Cartan, H.: Differential calculus. London: Kershaw Publishing 1971

20. Hörmander, L.: The analysis of linear partial operators III. Chap. XX. Berlin, Heidelberg, New York: Springer 1985

21. Hörmander, L.: Linear partial differential operators. Fourth Printing. Berlin, Heidelberg, New York: Springer 1976

22. Lopatinskiǐ, Ya.B.: Ukraǐn. Mat. Ž. 5, 123-151 (1953)

23. Săpiro, Ja.Z.: Isv. AN, ser. Matem. 17, 539-562 (1953)

24. McOwen, R.C.: Commun. Pure Appl. Math. 32, 783 (1979)

25. Lockhart, R.: Trans. Am. Math. Soc. 301, 1-35 (1987)

26. Lockhart, R., McOwen, R.C.: Ann. Scuola Norm. Sup. Pisa 22 (1985)

27. Cantor, M.: J. Diff. Eqns. 34, 102-113 (1979)

28. Gilbarg, D., Trudinger, N.S.: Elliptic partial differential equations of second order. Berlin, Heidelberg, New York: Springer 1977

29. Meyers, N.: J. Math. Mechs. 12, 247 (1963)

30. Simon, W.: Thesis, Univ. Wien (1980)

31. Martin, A.: Private communication

Communicated by S.-T. Yau

Received February 9, 1988; in revised form July 5, 1988 